Pacific

Journal of

Mathematics

\title{
EQUIVARIANT PRINCIPAL BUNDLES AND LOGARITHMIC CONNECTIONS ON TORIC VARIETIES
}

INDRANIL Biswas, ARIJIT Dey AND MaINAK PoddAR 


\title{
EQUIVARIANT PRINCIPAL BUNDLES AND LOGARITHMIC CONNECTIONS ON TORIC VARIETIES
}

\author{
INDRANIL BISWAS, ARIJIT DEY AND MAINAK PODDAR
}

Let $M$ be a smooth complex projective toric variety equipped with an action of a torus $T$, such that the complement $D$ of the open $T$-orbit in $M$ is a simple normal crossing divisor. Let $G$ be a complex reductive affine algebraic group. We prove that an algebraic principal $G$-bundle $E_{G} \rightarrow M$ admits a $T$-equivariant structure if and only if $E_{G}$ admits a logarithmic connection singular over $D$. If $E_{H} \rightarrow M$ is a $T$-equivariant algebraic principal $H$-bundle, where $H$ is any complex affine algebraic group, then $E_{H}$ in fact has a canonical integrable logarithmic connection singular over $D$.

\section{Introduction}

Our aim is to give characterizations of the equivariant principal bundles on smooth complex projective toric varieties.

Let $M$ be a smooth complex projective toric variety equipped with an action

$$
\rho: T \times M \rightarrow M
$$

of a torus $T$. For any point $t \in T$, define the automorphism

$$
\rho_{t}: M \rightarrow M, \quad x \mapsto \rho(t, x) .
$$

We assume that the complement $D$ of the open $T$-orbit in $M$ is a simple normal crossing divisor.

Let $G$ be a complex reductive affine algebraic group, and let $E_{G}$ be an algebraic principal $G$-bundle on $M$. In Proposition 4.1 we prove the following:

The principal $G$-bundle $E_{G}$ admits a T-equivariant structure if and only if the pulled-back principal G-bundle $\rho_{t}^{*} E_{G}$ is isomorphic to $E_{G}$ for every $t \in T$.

When $G=\operatorname{GL}(n, \mathbb{C})$, this result was proved by Klyachko [1989, p. 342, Proposition 1.2.1].

Biswas is supported by a J. C. Bose Fellowship. Poddar is supported by a FAPA grant from Universidad de los Andes.

MSC2010: primary 14L30, 14M27; secondary 14M17.

Keywords: smooth toric variety, logarithmic connection, equivariant principal bundle. 
Using the above characterization of $T$-equivariant principal $G$-bundles on $M$, we prove the following (see Theorem 4.2):

The principal G-bundle $E_{G}$ admits a logarithmic connection singular over $D$ if and only if $E_{G}$ admits a T-equivariant structure.

The "if" part of Theorem 4.2 does not require $G$ to be reductive. More precisely, any $T$-equivariant principal $H$-bundle $E_{H} \rightarrow M$, where $H$ is any complex affine algebraic group, admits a canonical integrable logarithmic connection singular over $D$ (see Proposition 3.2).

\section{Equivariant bundles}

Let $\mathbb{G}_{m}=\mathbb{C} \backslash\{0\}$ be the multiplicative group. Take a complex algebraic group $T$ which is isomorphic to a product of copies of $\mathbb{G}_{m}$. Let $M$ be a smooth irreducible complex projective variety equipped with an algebraic action of $T$

$$
\rho: T \times M \rightarrow M
$$

such that

- there is a Zariski open dense subset $M^{0} \subset M$ with $\rho\left(T, M^{0}\right)=M^{0}$,

- the action of $T$ on $M^{0}$ is free and transitive, and

- the complement $M \backslash M^{0}$ is a simple normal crossing divisor of $M$.

In particular, $M$ is a smooth projective toric variety. Note that $M^{0}$ is the unique $T$-orbit in $M$ with trivial isotropy.

Let $G$ be a connected complex affine algebraic group. A $T$-equivariant principal $G$-bundle on $M$ is a pair $\left(E_{G}, \tilde{\rho}\right)$, where

$$
p: E_{G} \rightarrow M
$$

is an algebraic principal $G$-bundle, and

$$
\tilde{\rho}: T \times E_{G} \rightarrow E_{G}
$$

is an algebraic action of $T$ on the total space of $E_{G}$, such that

- $p \circ \tilde{\rho}=\rho \circ\left(\operatorname{Id}_{T} \times p\right)$, where $\rho$ is the action in (2-1), and

- the actions of $T$ and $G$ on $E_{G}$ commute.

Fix a point $x_{0} \in M^{0} \subset M$. Let

$$
\iota: \rho\left(T, x_{0}\right)=M^{0} \hookrightarrow M
$$

be the inclusion map. Let $M^{0} \times G$ be the trivial principal $G$-bundle on $M^{0}$. It has a tautological integrable algebraic connection given by its trivialization. 
Let $\left(E_{G}, \tilde{\rho}\right)$ be a $T$-equivariant principal $G$-bundle on $M$. Fix a point $z_{0} \in\left(E_{G}\right)_{x_{0}}$. Using $z_{0}$, the action $\tilde{\rho}$ produces an isomorphism of principal $G$-bundles between $M^{0} \times G$ and the restriction $\left.E_{G}\right|_{M^{0}}$. This isomorphism of principal $G$-bundles is uniquely determined by the following two conditions:

- this isomorphism is $T$-equivariant (the action of $T$ on $M^{0} \times G$ is given by the action of $T$ on $M^{0}$ ), and

- it takes the point $z_{0} \in E_{G}$ to $\left(x_{0}, e\right) \in M^{0} \times G$.

Using this trivialization of $\left.E_{G}\right|_{M^{0}}$, the tautological integrable algebraic connection on $M^{0} \times G$ produces an integrable algebraic connection $\mathcal{D}^{0}$ on $\left.E_{G}\right|_{M^{0}}$. We note that the connection $\mathcal{D}^{0}$ is independent of the choice of the points $x_{0}$ and $z_{0}$. Indeed, the flat sections for $\mathcal{D}^{0}$ are precisely the orbits of $T$ in $\left.E_{G}\right|_{M^{0}}$. Note that this description of $\mathcal{D}^{0}$ does not require choosing base points in $M^{0}$ and $\left.E_{G}\right|_{M^{0}}$.

In Proposition 3.2 it will be shown that $\mathcal{D}^{0}$ extends to a logarithmic connection on $E_{G}$ over $M$ singular over the simple normal crossing divisor $M \backslash M^{0}$.

\section{Logarithmic connections}

A canonical trivialization. The Lie algebra of $T$ will be denoted by $\mathfrak{t}$. Let

$$
\mathcal{V}:=M \times \mathfrak{t} \rightarrow M
$$

be the trivial vector bundle with fiber $t$. The holomorphic tangent bundle of $M$ will be denoted by $T M$. Consider the action of $T$ on $M$ in (2-1). It produces a homomorphism of $\mathcal{O}_{M}$-coherent sheaves

$$
\beta: \mathcal{V} \rightarrow T M
$$

Let

$$
D:=M \backslash M^{0}
$$

be the simple normal crossing divisor of $M$. Let

$$
T M(-\log D) \subset T M
$$

be the corresponding logarithmic tangent bundle. Recall that $T M(-\log D)$ is characterized as the maximal coherent subsheaf of $T M$ that preserves $\mathcal{O}_{M}(-D) \subset \mathcal{O}_{M}$ for the derivation action of $T M$ on $\mathcal{O}_{M}$.

\section{Lemma 3.1.}

(1) The image of $\beta$ in (3-2) is contained in the subsheaf $T M(-\log D) \subset T M$.

(2) The resulting homomorphism $\beta: \mathcal{V} \rightarrow T M(-\log D)$ is an isomorphism.

Proof. The divisor $D$ is preserved by the action of $T$ on $M$. Therefore, the action 
of $T$ on $\mathcal{O}_{M}$, given by the action of $T$ on $M$, preserves the subsheaf $\mathcal{O}_{M}(-D)$. From this it follows immediately that the subsheaf $\mathcal{O}_{M}(-D) \subset \mathcal{O}_{M}$ is preserved by the derivation action of the subsheaf

$$
\beta(\mathcal{V}) \subset T M .
$$

Therefore, we conclude that $\beta(\mathcal{V}) \subset T M(-\log D)$.

It is known that the vector bundle $T M(-\log D)$ is holomorphically trivial. This follows from Proposition 2 in [Fulton 1993, p. 87], which says that $\Omega_{M}^{1}(\log D)$ is holomorphically trivial, together with the equality $\Omega_{M}^{1}(\log D)^{*}=T M(-\log D)$.

So, both $\mathcal{V}$ and $T M(-\log D)$ are trivial vector bundles, and $\beta$ is a homomorphism between them which is an isomorphism over the open subset $M^{0}$. From this it can be deduced that $\beta$ is an isomorphism over entire $M$. To see this, consider the homomorphism

$$
\bigwedge^{r} \beta: \bigwedge^{r} \mathcal{V} \rightarrow \bigwedge^{r} T M(-\log D)
$$

induced by $\beta$, where $r=\operatorname{dim}_{\mathbb{C}} T=\operatorname{rank}(\mathcal{V})$. So $\bigwedge^{r} \beta$ is a holomorphic section of the line bundle $\left(\wedge^{r} T M(-\log D)\right) \otimes\left(\bigwedge^{r} \mathcal{V}\right)^{*}$. This line bundle is holomorphically trivial because both $\mathcal{V}$ and $T M(-\log D)$ are holomorphically trivial. Fixing a trivialization of $\left(\wedge^{r} T M(-\log D)\right) \otimes\left(\bigwedge^{r} \mathcal{V}\right)^{*}$, we consider $\wedge^{r} \beta$ as a holomorphic function on $M$. This function is nowhere vanishing because it does not vanish on $M^{0}$ and holomorphic functions on $M$ are constants. Since $\bigwedge^{r} \beta$ is nowhere vanishing, the homomorphism $\beta$ is an isomorphism.

A canonical logarithmic connection on equivariant bundles. The Lie algebra of $G$ will be denoted by $\mathfrak{g}$.

Let $p: E_{G} \rightarrow M$ be an algebraic principal $G$-bundle. Consider the differential

$$
d p: T E_{G} \rightarrow p^{*} T M,
$$

where $T E_{G}$ is the algebraic tangent bundle of $E_{G}$. The kernel of $d p$ will be denoted by $T_{E_{G}} / M$. Using the action of $G$ on $E_{G}$, the subbundle $T_{E_{G} / M} \subset T E_{G}$ is identified with the trivial vector bundle over $E_{G}$ with fiber $\mathfrak{g}$.

The action of $G$ on $E_{G}$ produces an action of $G$ on $T E_{G}$. So we get an action of $G$ on the quasicoherent sheaf $p_{*} T E_{G}$ on $M$. The invariant part

$$
\operatorname{At}\left(E_{G}\right):=\left(p_{*} T E_{G}\right)^{G} \subset p_{*} T E_{G}
$$

is a locally free coherent sheaf; its coherence property follows from the fact that the action of $G$ on the fibers of $p$ is transitive, implying that a $G$-invariant section of $\left.\left(T E_{G}\right)\right|_{p^{-1}(x)}, x \in M$, is uniquely determined by its evaluation at just one point of the fiber $p^{-1}(x)$. Also note that $\operatorname{At}\left(E_{G}\right)=\left(T E_{G}\right) / G$. This $\operatorname{At}\left(E_{G}\right)$ is known as the Atiyah bundle for $E_{G}$. Since $T_{E_{G} / M}$ is identified with $E_{G} \times \mathfrak{g}$, the invariant 
direct image $\left(p_{*} T_{E_{G} / M}\right)^{G}$ is identified with the adjoint vector bundle

$$
\operatorname{ad}\left(E_{G}\right):=E_{G} \times{ }^{G} \mathfrak{g} \rightarrow M
$$

associated to $E_{G}$ for the adjoint action of $G$ on $\mathfrak{g}$. We note that $\operatorname{ad}\left(E_{G}\right)=T_{E_{G}} / M / G$. Now the differential $d p$ in (3-4) produces a short exact sequence of holomorphic vector bundles on $M$

$$
0 \rightarrow \operatorname{ad}\left(E_{G}\right) \rightarrow \operatorname{At}\left(E_{G}\right) \stackrel{\phi}{\longrightarrow} T M \rightarrow 0,
$$

which is known as the Atiyah exact sequence. A holomorphic connection on $E_{G}$ over $M$ is a holomorphic splitting

$$
T M \rightarrow \operatorname{At}\left(E_{G}\right)
$$

of (3-5) [Atiyah 1957].

As before, setting $D=M \backslash M^{0}$, define

$$
\operatorname{At}\left(E_{G}\right)(-\log D):=\phi^{-1}(T M(-\log D)) \subset \operatorname{At}\left(E_{G}\right),
$$

where $\phi$ is the projection in (3-5) and $T M(-\log D)$ is the subsheaf in (3-3). So (3-5) gives the following short exact sequence of holomorphic vector bundles on $M$ :

$$
0 \rightarrow \operatorname{ad}\left(E_{G}\right) \rightarrow \operatorname{At}\left(E_{G}\right)(-\log D) \stackrel{\phi}{\longrightarrow} T M(-\log D) \rightarrow 0 .
$$

A logarithmic connection on $E_{G}$, with singular locus $D$, is a holomorphic homomorphism

$$
\delta: T M(-\log D) \rightarrow \operatorname{At}\left(E_{G}\right)(-\log D)
$$

such that $\phi \circ \delta$ is the identity automorphism of $T M(-\log D)$, where $\phi$ is the homomorphism in (3-6). Just like the curvature of a connection, the curvature of a logarithmic connection $\delta$ on $E_{G}$ is the obstruction for the homomorphism $\delta$ to preserve the Lie algebra structure of the sheaf of sections of $T M(-\log D)$ and $\operatorname{At}\left(E_{G}\right)(-\log D)$ given by the Lie bracket of vector fields. In particular, $\delta$ is called integrable (or flat) if it preserves the Lie algebra structure of the sheaf of sections of $T M(-\log D)$ and $\operatorname{At}\left(E_{G}\right)(-\log D)$ given by the Lie bracket of vector fields.

Proposition 3.2. Let $\left(E_{G}, \tilde{\rho}\right)$ be a T-equivariant principal $G$-bundle on $M$. Then $E_{G}$ admits an integrable logarithmic connection that restricts to the connection $\mathcal{D}^{0}$ on $M^{0}$ constructed in Section 2.

Proof. Let

$$
\tilde{\mathcal{V}}:=E_{G} \times \mathfrak{t} \rightarrow E_{G}
$$

be the trivial vector bundle over $E_{G}$ with fiber $\mathrm{t}$. Note that $p^{*} \mathcal{V}=\tilde{\mathcal{V}}$, where $\mathcal{V}$ is the vector bundle in (3-1), and $p$, as before, is the projection of $E_{G}$ to $M$. 
The action $\tilde{\rho}$ of $T$ on $E_{G}$ produces a homomorphism

$$
\tilde{\beta}: \tilde{\mathcal{V}} \rightarrow T E_{G} .
$$

Since $p^{-1}(D)$ is preserved by the action of $T$ on $E_{G}$, the induced action of $T$ on $\mathcal{O}_{E_{G}}$ preserves the subsheaf $\mathcal{O}_{E_{G}}\left(-p^{-1}(D)\right)$. Hence the image of $\tilde{\beta}$ lies inside the subsheaf

$$
T E_{G}\left(-\log p^{-1}(D)\right) \subset T E_{G} .
$$

Note that $p^{-1}(D)$ is a simple normal crossing divisor on $E_{G}$ because $D$ is a simple normal crossing divisor on $M$.

In Lemma 3.1(2) we saw that $\beta$ is an isomorphism. Consider

$$
p^{*} \beta^{-1}: p^{*}(T M(-\log D)) \rightarrow p^{*} \mathcal{V}=\tilde{\mathcal{V}} .
$$

Precomposing this with $\tilde{\beta}$ in (3-7), we have

$$
\tilde{\beta} \circ\left(p^{*} \beta^{-1}\right): p^{*}(T M(-\log D)) \rightarrow T E_{G}\left(-\log p^{-1}(D)\right) .
$$

We observe that the homomorphism $\tilde{\beta} \circ\left(p^{*} \beta^{-1}\right)$ is $G$-equivariant for the trivial action of $G$ on $p^{*}(T M(-\log D))$ and the action of $G$ on $T E_{G}\left(-\log p^{-1}(D)\right)$ induced by the action of $G$ on $E_{G}$. Therefore, taking the $G$-invariant parts of the direct images by $p$, the above homomorphism $\tilde{\beta} \circ\left(p^{*} \beta^{-1}\right)$ produces a homomorphism

$$
\begin{aligned}
\beta^{\prime}: T M(-\log D)=\left(p_{*} p^{*}(T M\right. & (-\log D)))^{G} \\
& \rightarrow\left(p_{*} T E_{G}\left(-\log p^{-1}(D)\right)\right)^{G}=\operatorname{At}\left(E_{G}\right)(-\log D) .
\end{aligned}
$$

It is now straightforward to check that the homomorphism $\beta^{\prime}$ produces a holomorphic splitting of the exact sequence in (3-6). Therefore, $\beta^{\prime}$ defines a logarithmic connection on $E_{G}$ singular on $D$. The restriction of this logarithmic connection to $M^{0}$ clearly coincides with the connection $\mathcal{D}^{0}$ constructed in Section 2.

\section{A criterion for equivariance}

For each point $t \in T$, define the automorphism

$$
\rho_{t}: M \rightarrow M, \quad x \mapsto \rho(t, x),
$$

where $\rho$ is the action in (2-1). If $\left(E_{G}, \tilde{\rho}\right)$ is a $T$-equivariant principal $G$-bundle on $M$, then clearly the map

$$
E_{G} \rightarrow E_{G}, \quad z \mapsto \tilde{\rho}(t, z)
$$

is an isomorphism of the principal $G$-bundle $\rho_{t}^{*} E_{G}$ with $E_{G}$. The aim in this section is to prove a converse of this statement. 
Take an algebraic principal $G$-bundle

$$
p: E_{G} \rightarrow M
$$

Let $\mathcal{G}$ be the set of all pairs of the form $(t, f)$, where $t \in T$ and where

$$
f: E_{G} \rightarrow E_{G}
$$

is an algebraic automorphism of the variety $E_{G}$ satisfying the following conditions:

- $p \circ f=\rho_{t} \circ p$, and

- $f$ intertwines the action of $G$ on $E_{G}$.

Note that the above two conditions imply that $f$ is an algebraic isomorphism of the principal $G$-bundle $\rho_{t}^{*} E_{G}$ with $E_{G}$.

We have the following composition on the set $\mathcal{G}$ :

$$
\left(t_{1}, f_{1}\right) \cdot\left(t_{2}, f_{2}\right):=\left(t_{1} \circ t_{2}, f_{1} \circ f_{2}\right) .
$$

The inverse of $(t, f)$ is $\left(t^{-1}, f^{-1}\right)$. These operations make $\mathcal{G}$ a group. In fact, $\mathcal{G}$ has the structure of an affine algebraic group defined over $\mathbb{C}$. Let $\mathcal{A}$ denote the group of all algebraic automorphisms of the principal $G$-bundle $E_{G}$. So $\mathcal{A}$ is a subgroup of $\mathcal{G}$ with the inclusion map being $f \mapsto(e, f)$. We have a natural projection

$$
h: \mathcal{G} \rightarrow T, \quad(t, f) \mapsto t
$$

which fits in the following exact sequence of complex affine algebraic groups:

$$
0 \rightarrow \mathcal{A} \rightarrow \mathcal{G} \stackrel{h}{\longrightarrow} T \text {. }
$$

We note that there is a tautological action of $\mathcal{G}$ on $E_{G}$; the action of any $(t, f) \in \mathcal{G}$ on $E_{G}$ is given by the map defined by $y \mapsto f(y)$.

Now assume that $E_{G}$ satisfies the condition that, for every $t \in T$, the pulled-back principal $G$-bundle $\rho_{t}^{*} E_{G}$ is isomorphic to $E_{G}$. This assumption is equivalent to the statement that the homomorphism $h$ in (4-1) is surjective.

In view of the above assumption, the sequence in (4-1) becomes the following short exact sequence of complex affine algebraic groups:

$$
0 \rightarrow \mathcal{A} \rightarrow \mathcal{G} \stackrel{h}{\longrightarrow} T \rightarrow 0 .
$$

Let $\mathcal{G}^{0} \subset \mathcal{G}$ be the connected component containing the identity element. Since $T$ is connected and $h$ is surjective, the restriction of $h$ to $\mathcal{G}^{0}$ is also surjective. Therefore, from (4-2) we have the short exact sequence of affine complex algebraic groups

$$
0 \rightarrow \mathcal{A}^{0} \stackrel{\mathfrak{A}}{\longrightarrow} \mathcal{G}^{0} \stackrel{h^{0}}{\longrightarrow} T \rightarrow 0,
$$

where $\mathcal{A}^{0}:=\mathcal{A} \cap \mathcal{G}^{0}$ and $h^{0}:=\left.h\right|_{\mathcal{G}^{0}}$. 
Take a maximal torus $T_{\mathcal{G}} \subset \mathcal{G}^{0}$. From (4-3) it follows that the restriction

$$
h^{\prime}:=\left.h^{0}\right|_{T_{\mathcal{G}}}: T_{\mathcal{G}} \rightarrow T
$$

is surjective. Define $T_{\mathcal{A}}:=\mathcal{A}^{0} \cap T_{\mathcal{G}} \subset T_{\mathcal{G}}$ using the homomorphism $\iota_{\mathcal{A}}$ in (4-3). Therefore, from (4-3) we have the short exact sequence of algebraic groups

$$
0 \rightarrow T_{\mathcal{A}} \stackrel{\mathfrak{L A}_{\mathcal{A}} \mid T_{\mathcal{A}}}{\longrightarrow} T_{\mathcal{G}} \stackrel{h^{\prime}}{\longrightarrow} T \rightarrow 0 .
$$

Recall that $\mathcal{G}$ has a tautological action on $E_{G}$. Therefore, the subgroup $T_{\mathcal{G}}$ has a tautological action on $E_{G}$ which is the restriction of the tautological action of $\mathcal{G}$.

Now we assume that the group $G$ is reductive.

A parabolic subgroup of $G$ is a connected Zariski closed subgroup $P \subset G$ such that the variety $G / P$ is projective. For a parabolic subgroup $P$, its unipotent radical will be denoted by $R_{u}(P)$. A Levi subgroup of $P$ is a connected reductive subgroup $L(P) \subset P$ such that the composition

$$
L(P) \hookrightarrow P \rightarrow P / R_{u}(P)
$$

is an isomorphism. Levi subgroups exist, and any two Levi subgroups of $P$ differ by conjugation by an element of $R_{u}(P)$ [Humphreys 1975, p. 184-185, §30.2; Borel 1991, p. 158, 11.22 and 11.23].

Let $\operatorname{Ad}\left(E_{G}\right):=E_{G} \times{ }^{G} G \rightarrow M$ be the adjoint bundle associated to $E_{G}$ for the adjoint action of $G$ on itself. The fibers of $\operatorname{Ad}\left(E_{G}\right)$ are groups identified with $G$ up to an inner automorphism; the corresponding Lie algebra bundle is $\operatorname{ad}\left(E_{G}\right)$. We note that $\mathcal{A}$ in (4-2) is the space of all algebraic sections of $\operatorname{Ad}\left(E_{G}\right)$.

Using the action of $T_{\mathcal{A}}$ on $E_{G}$, we have

- a Levi subgroup $L(P)$ of a parabolic subgroup $P$ of $G$, and

- an algebraic reduction of structure group $E_{L(P)} \subset E_{G}$ of $E_{G}$ to $L(P)$ which is preserved by the tautological action of $T_{\mathcal{G}}$ on $E_{G}$,

such that the image of $T_{\mathcal{A}}$ in $\operatorname{Ad}\left(E_{G}\right)$ (recall that the elements of $\mathcal{A}$ are sections of $\left.\operatorname{Ad}\left(E_{G}\right)\right)$ lies in the connected component, containing the identity element, of the center of each fiber of $\operatorname{Ad}\left(E_{L(P)}\right) \subset \operatorname{Ad}\left(E_{G}\right)$ (see [Balaji et al. 2005; Biswas and Parameswaran 2006] for the construction of $\left.E_{L(P)}\right)$. The construction of $E_{L(P)}$ requires fixing a point $z_{0}$ of $E_{G}$, where $E_{L(P)}$ contains $z_{0}$. Using $z_{0}$, the fiber $\left(E_{L(P)}\right)_{p\left(z_{0}\right)}$ is identified with $L(P)$. Moreover, the evaluation, at $p\left(z_{0}\right)$, of the sections of $\operatorname{Ad}\left(E_{G}\right)$ corresponding to the elements of $T_{\mathcal{A}}$ makes $T_{\mathcal{A}}$ a subgroup of the connected component, containing the identity element, of the center of $E_{L(P)}$; in particular, this evaluation map on $T_{\mathcal{A}}$ is injective (see the second paragraph in [Balaji et al. 2005, p. 230, Section 3]). We briefly recall (from [Balaji et al. 2005; Biswas and Parameswaran 2006]) the argument that the evaluation map on semisimple elements of $\mathcal{A}$ is injective. Let $\xi$ be a semisimple element of $\mathcal{A}=\Gamma\left(M, \operatorname{Ad}\left(E_{G}\right)\right)$. 
Since $\xi$ is semisimple, for each point $x \in M$, the evaluation $\xi(x)$ is a semisimple element of $\left.\operatorname{Ad}\left(E_{G}\right)\right)_{x}$. The group $\left.\operatorname{Ad}\left(E_{G}\right)\right)_{x}$ is identified with $G$ up to an inner automorphism of $G$. All conjugacy classes of a semisimple element of $G$ are parametrized by $T_{G} / W_{T_{G}}$, where $T_{G}$ is a maximal torus in $G$, and $W_{T_{G}}=N\left(T_{G}\right) / T_{G}$ is the Weyl group with $N\left(T_{G}\right)$ being the normalizer of $T_{G}$ in $G$. We note that $T_{G} / W_{T_{G}}$ is an affine variety. Therefore, we get a morphism $\xi^{\prime}: M \rightarrow T_{G} / W_{T_{G}}$ that sends any $x \in M$ to the conjugacy class of $\xi(x)$. Since $M$ is a projective variety and $T_{G} / W_{T_{G}}$ is an affine variety, we conclude that $\xi^{\prime}$ is a constant map. So if $\xi(x)=e$ for some $x \in M$, then $\xi=e$ identically.

Let $Z_{L(P)}^{0} \subset L(P)$ be the connected component, containing the identity element, of the center. We note that $Z_{L(P)}^{0}$ is a product of copies of $\mathbb{G}_{m}$. Therefore, the above injective homomorphism $T_{\mathcal{A}} \rightarrow Z_{L(P)}^{0}$ extends to a homomorphism

$$
\eta: T_{\mathcal{G}} \rightarrow Z_{L(P)}^{0} .
$$

Define

$$
\eta^{\prime}:=\tau \circ \eta
$$

where $\tau$ is the inversion homomorphism of $Z_{L(P)}^{0}$ defined by $g \mapsto g^{-1}$.

Consider the action of $T_{\mathcal{G}}$ on $E_{L(P)}$; recall that $E_{L(P)}$ is preserved by the tautological action of $T_{\mathcal{G}}$ on $E_{G}$. We can twist this action on $E_{L(P)}$ by $\eta^{\prime}$ in (4-5), because the actions of $Z_{L(P)}^{0}$ and $L(P)$ on $E_{L(P)}$ commute. For this new action, the group $T_{\mathcal{A}}$ clearly acts trivially on $E_{L(P)}$.

Consider the above action of $T_{\mathcal{G}}$ on $E_{L(P)}$ constructed using $\eta^{\prime}$. Since $T_{\mathcal{A}}$ acts trivially on $E_{L(P)}$, the action of $T_{\mathcal{G}}$ on $E_{L(P)}$ descends to an action of $T$ on $E_{L(P)}$ (see (4-4)). The principal $G$-bundle $E_{G}$ is the extension of the structure group of $E_{L(P)}$ using the inclusion of $L(P)$ in $G$. Therefore, the above action of $T$ on $E_{L(P)}$ produces an action of $T$ on $E_{G}$. More precisely, the total space of $E_{G}$ is the quotient of $E_{L(P)} \times G$ where two elements $\left(z_{1}, g_{1}\right)$ and $\left(z_{2}, g_{2}\right)$ of $E_{L(P)} \times G$ are identified if there is an element $g \in L(P)$ such that $z_{2}=z_{1} g$ and $g_{2}=g^{-1} g_{1}$. Now the action of $T$ on $E_{L(P)} \times G$, given by the above action of $T$ on $E_{L(P)}$ and the trivial action of $T$ on $G$, descends to an action of $T$ on the quotient space $E_{G}$. Consequently, $E_{G}$ admits a $T$-equivariant structure.

Therefore, we have proved the following:

Proposition 4.1. Let $G$ be reductive, and let $E_{G} \rightarrow M$ be a principal $G$-bundle such that, for every $t \in T$, the pulled-back principal G-bundle $\rho_{t}^{*} E_{G}$ is isomorphic to $E_{G}$. Then $E_{G}$ admits a T-equivariant structure.

For vector bundles on $M$, Proposition 4.1 was proved by Klyachko [1989, p. 342, Proposition 1.2.1]. 


\section{Equivariance property from a logarithmic connection.}

Theorem 4.2. Let $G$ be reductive, and let $p: E_{G} \rightarrow M$ be a principal $G$-bundle admitting a logarithmic connection whose singularity locus is contained in the divisor $D=M \backslash M^{0}$. Then $E_{G}$ admits a T-equivariant structure.

Proof. Since $E_{G}$ admits a logarithmic connection, by definition, there is a homomorphism of coherent sheaves

$$
\delta: T M(-\log D) \rightarrow \operatorname{At}\left(E_{G}\right)(-\log D)
$$

such that $\phi \circ \delta$ is the identity automorphism of $T M(-\log D)$, where $\phi$ is the homomorphism in (3-6). Let

$$
\hat{\delta}: H^{0}(M, T M(-\log D)) \rightarrow H^{0}\left(M, \operatorname{At}\left(E_{G}\right)(-\log D)\right)
$$

be the homomorphism of global sections given by $\delta$. From Lemma 3.1(2) we know that $H^{0}(M, T M(-\log D))$ is the Lie algebra $\mathfrak{t}$ of $T$.

We will now show that there is a natural injective homomorphism

$$
\theta: H^{0}\left(M, \operatorname{At}\left(E_{G}\right)(-\log D)\right) \rightarrow \operatorname{Lie}(\mathcal{G}),
$$

where $\operatorname{Lie}(\mathcal{G})$ is the Lie algebra of the group $\mathcal{G}$ in (4-1).

The elements of $\operatorname{Lie}(\mathcal{G})$ are all holomorphic sections $s \in H^{0}\left(M, \operatorname{At}\left(E_{G}\right)\right)$ such that the vector field $\phi(s)$, where $\phi$ is the projection in (3-5), is of the form $\beta\left(s^{\prime}\right)$, where $s^{\prime} \in \mathfrak{t}$ and where $\beta$ is the homomorphism in (3-2). Now, if

$$
s \in H^{0}\left(M, \operatorname{At}\left(E_{G}\right)(-\log D)\right) \subset H^{0}\left(M, \operatorname{At}\left(E_{G}\right)\right),
$$

then $\phi(s)$ is a holomorphic section of $T M(-\log D)$ (see (3-6)). From Lemma 3.1(2) it now follows that $\phi(s)$ is of the form $\beta\left(s^{\prime}\right)$, where $s^{\prime} \in \mathfrak{t}$. This gives us the injective homomorphism in (4-6).

Finally, consider the composition

$$
\theta \circ \hat{\delta}: \mathfrak{t}=H^{0}(M, T M(-\log D)) \rightarrow \operatorname{Lie}(\mathcal{G}) .
$$

From its construction it follows that

$$
(d h) \circ \theta \circ \hat{\delta}=\operatorname{Id}_{\mathfrak{t}},
$$

where $d h: \operatorname{Lie}(\mathcal{G}) \rightarrow \mathfrak{t}$ is the homomorphism of Lie algebras given by $h$ in (4-1). In particular, $d h$ is surjective. Since $T$ is connected, this immediately implies that the homomorphism $h$ is surjective. Now from Proposition 4.1 it follows that $E_{G}$ admits a $T$-equivariant structure. 


\section{References}

[Atiyah 1957] M. F. Atiyah, "Complex analytic connections in fibre bundles", Trans. Amer. Math. Soc. 85 (1957), 181-207. MR 19,172c Zbl 0078.16002

[Balaji et al. 2005] V. Balaji, I. Biswas, and D. S. Nagaraj, "Krull-Schmidt reduction for principal bundles", J. Reine Angew. Math. 578 (2005), 225-234. MR 2006e:14061 Zbl 1084.14041

[Biswas and Parameswaran 2006] I. Biswas and A. J. Parameswaran, "On the equivariant reduction of structure group of a principal bundle to a Levi subgroup", J. Math. Pures Appl. (9) 85:1 (2006), 54-70. MR 2006k:14081 Zbl 1159.14307

[Borel 1991] A. Borel, Linear algebraic groups, 2nd ed., Graduate Texts in Mathematics 126, Springer, New York, 1991. MR 92d:20001 Zbl 0726.20030

[Fulton 1993] W. Fulton, Introduction to toric varieties, Annals of Mathematics Studies 131, Princeton Univ. Press, 1993. MR 94g:14028 Zbl 0813.14039

[Humphreys 1975] J. E. Humphreys, Linear algebraic groups, Graduate Texts in Mathematics 21, Springer, New York, 1975. MR 53 \#633 Zbl 0325.20039

[Klyachko 1989] A. A. Klyachko, "Equivariant bundles on toral varieties", Izv. Akad. Nauk SSSR Ser. Mat. 53:5 (1989), 1001-1039. In Russian; translated in Math. USSR Izv. 35:2 (1990), 337-375. MR 91c:14064 Zbl 0706.14010

Received March 11, 2015. Revised June 8, 2015.

INDRANIL BISWAS

SCHOOL OF MATHEMATICS

TATA Institute of Fundamental RESEARCH

HOMI BHABHA ROAD

MUMBAI 400005

INDIA

indranil@math.tifr.res.in

ARIJIT DEY

DEPARTMENT OF MATHEMATICS

INDIAN INSTITUTE OF TECHNOLOGY MADRAS

CHENNAI 600036

INDIA

arijitdey@gmail.com

MAINAK PODDAR

DEPARTAMENTo de Matemáticas

UNIVERSIDAD DE LOS ANDES

BOGOTA

Colombia

mainakp@gmail.com 


\title{
PACIFIC JOURNAL OF MATHEMATICS
}

\author{
msp.org/pjm
}

Founded in 1951 by E. F. Beckenbach (1906-1982) and F. Wolf (1904-1989)

\section{EDITORS}

Don Blasius (Managing Editor)

Department of Mathematics

University of California

Los Angeles, CA 90095-1555

blasius@math.ucla.edu

\author{
Paul Balmer \\ Department of Mathematics \\ University of California \\ Los Angeles, CA 90095-1555 \\ balmer@math.ucla.edu \\ Robert Finn \\ Department of Mathematics \\ Stanford University \\ Stanford, CA 94305-2125 \\ finn@math.stanford.edu \\ Sorin Popa \\ Department of Mathematics \\ University of California \\ Los Angeles, CA 90095-1555 \\ popa@math.ucla.edu
}

\author{
Vyjayanthi Chari \\ Department of Mathematics \\ University of California \\ Riverside, CA 92521-0135 \\ chari@math.ucr.edu \\ Kefeng Liu \\ Department of Mathematics \\ University of California \\ Los Angeles, CA 90095-1555 \\ liu@math.ucla.edu \\ Jie Qing \\ Department of Mathematics \\ University of California \\ Santa Cruz, CA 95064 \\ qing@ cats.ucsc.edu
}

\section{PRODUCTION}

Silvio Levy, Scientific Editor, production@msp.org

\section{SUPPORTING INSTITUTIONS}

ACADEMIA SINICA, TAIPEI

CALIFORNIA INST. OF TECHNOLOGY

INST. DE MATEMÁTICA PURA E APLICADA

KEIO UNIVERSITY

MATH. SCIENCES RESEARCH INSTITUTE

NEW MEXICO STATE UNIV.

OREGON STATE UNIV.

\author{
STANFORD UNIVERSITY \\ UNIV. OF BRITISH COLUMBIA \\ UNIV. OF CALIFORNIA, BERKELEY \\ UNIV. OF CALIFORNIA, DAVIS \\ UNIV. OF CALIFORNIA, LOS ANGELES \\ UNIV. OF CALIFORNIA, RIVERSIDE \\ UNIV. OF CALIFORNIA, SAN DIEGO \\ UNIV. OF CALIF., SANTA BARBARA
}

\author{
Daryl Cooper \\ Department of Mathematics \\ University of California \\ Santa Barbara, CA 93106-3080 \\ cooper@math.ucsb.edu \\ Jiang-Hua Lu \\ Department of Mathematics \\ The University of Hong Kong \\ Pokfulam Rd., Hong Kong \\ jhlu@maths.hku.hk \\ Paul Yang \\ Department of Mathematics \\ Princeton University \\ Princeton NJ 08544-1000 \\ yang@math.princeton.edu
}

These supporting institutions contribute to the cost of publication of this Journal, but they are not owners or publishers and have no responsibility for its contents or policies.

See inside back cover or msp.org/pjm for submission instructions.

The subscription price for 2016 is US $\$ 440 /$ year for the electronic version, and $\$ 600 /$ year for print and electronic.

Subscriptions, requests for back issues and changes of subscribers address should be sent to Pacific Journal of Mathematics, P.O. Box 4163, Berkeley, CA 94704-0163, U.S.A. The Pacific Journal of Mathematics is indexed by Mathematical Reviews, Zentralblatt MATH, PASCAL CNRS Index, Referativnyi Zhurnal, Current Mathematical Publications and Web of Knowledge (Science Citation Index).

The Pacific Journal of Mathematics (ISSN 0030-8730) at the University of California, c/o Department of Mathematics, 798 Evans Hall \#3840, Berkeley, CA 94720-3840, is published twelve times a year. Periodical rate postage paid at Berkeley, CA 94704, and additional mailing offices. POSTMASTER: send address changes to Pacific Journal of Mathematics, P.O. Box 4163, Berkeley, CA 94704-0163.

PJM peer review and production are managed by EditFLOW ${ }^{\circledR}$ from Mathematical Sciences Publishers.

\section{PUBLISHED BY}

\section{mathematical sciences publishers \\ nonprofit scientific publishing}

http://msp.org/

(C) 2016 Mathematical Sciences Publishers 


\section{PACIFIC JOURNAL OF MATHEMATICS}

Volume $280 \quad$ No. $2 \quad$ February 2016

Topological Molino's theory

JESÚS A. ÁlVAREz LóPEZ and MANUEl F. MoreIRA GALICIA

Equivariant principal bundles and logarithmic connections on toric varieties

INDRANIL BISWAS, ARIJIT DEY and MAINAK PODDAR

On a spectral theorem in paraorthogonality theory

Kenier CASTILlo, RuYmán CRUZ-BARRoso and Francisco

PERDOMO-PÍO

Sigma theory and twisted conjugacy, II: Houghton groups and pure

symmetric automorphism groups

DACIBERG L. GONÇALVES and PARAMESWARAN SANKARAN

The second CR Yamabe invariant

PAK TUNG Ho

No hyperbolic pants for the 4-body problem with strong potential

CONNOR JACKMAN and RICHARD MONTGOMERY

Unions of Lebesgue spaces and $A_{1}$ majorants

Greg Knese, John E. M ${ }^{\mathrm{C}}$ CARThy and Kabe Moen

Complex hyperbolic $(3,3, n)$ triangle groups

JOHN R. PARKER, JiEYAN WANG and BAOHUA XIE

Topological aspects of holomorphic mappings of hyperquadrics from $\mathbb{C}^{2}$ to $\mathbb{C}^{3}$

\section{MICHAEL REITER}

2-Blocks with minimal nonabelian defect groups III

BENJAMIN SAMBALE

Number of singularities of stable maps on surfaces 